\title{
OPEN PROBLEMS ON BACKWARD STOCHASTIC DIFFERENTIAL EQUATIONS *
}

Shige Peng

School of Mathematics and System Science Shandong University, 250100, Jinan, China

\section{BASIC RESULTS ON BACKWARD STOCHASTIC DIFFERENTIAL EQUATIONS}

Recent years, many interesting problems in the theory of backward stochastic differential equations (in short, BSDE) have been solved. Others still remain open. In this paper, we will discuss those related to the stochastic control theory.

Let $(\Omega, \mathcal{F}, P)$ be a probability space, let $W_{t}, t \geq 0$ be a standard Brownnian motion. For the sake of simplification, $W_{t}$ is of 1-dimension. We are also limited to the interval $[0, T]$, with a finite constant $T>0$. (see Peng [1991], Duffie, Ma and Yong [1994], Hu [1995], Chen [1997], Peng and Shi [1998] for infinite horizon cases). All processes are assumed to be $\mathcal{F}_{t}$-adapted. Here the filtration $\left\{\mathcal{F}_{t}\right\}_{t \geq 0}$ is the natural one generated by the Brownian motion $W$. It is the information we have possessed up to time $t$. We will denote by $M^{2}\left(0, T ; \mathbf{R}^{n}\right)$ the set of all $\mathbf{R}^{n}$-valued square-integrable $\mathcal{F}_{t}$-adapted processes.

We are given $\xi$, an $\mathcal{F}_{T}$-measurable $\mathbf{R}^{m}$-valued square-integrable random vector, and a function $f(y, z, t, \omega): \mathbf{R}^{m} \times \mathbf{R}^{m} \times[0, T] \times \Omega \longmapsto \mathbf{R}^{m}$ such that, for each $(y, z) \in \mathbf{R}^{m} \times \mathbf{R}^{m}, f(x, y, \cdot) \in M^{2}\left(0, T ; \mathbf{R}^{m}\right)$. The problem of backward stochastic differential equation is to look for a pair of processes $(y ., z.) \in M^{2}\left(0, T ; \mathbf{R}^{m}\right)^{2}$, that solves

$$
\begin{aligned}
-d y_{t} & =f\left(y_{t}, z_{t}, t\right) d t-z_{s} d W_{t}, \quad t \in[0, T] \\
y_{T} & =\xi
\end{aligned}
$$

\footnotetext{
*This work was supported by NSF of China.
} 
Bismut [1973] studied the case when $f$ is linear in $(y, z)$. Pardoux and Peng [1990] obtained a general result: if $f$ is Lipschitz in $(y, z)$, then the solution $\left(y_{t}, z_{t}\right)_{0 \leq t \leq T}$ of BSDE (1.1) exists and is unique.

Peng [1991a] observed a mutual relation between the solution $u(x, t): \mathbf{R}^{n} \times$ $[0, T] \longmapsto \mathbf{R}^{m}$ of the the system of parabolic PDE

$$
\begin{aligned}
-\frac{\partial u^{k}}{\partial t} & =\frac{1}{2} \sum_{i, j=1}^{n}\left[\sigma \sigma^{T}\right]_{i j} \frac{\partial^{2} u^{k}}{\partial x_{i} \partial x_{j}}+\sum_{i=1}^{n} b_{i} \frac{\partial u^{k}}{\partial x_{i}}+f(u, \nabla u \sigma, x), \\
u^{k}(x, T) & =\Phi^{k}(x) \quad k=1, \cdots, m .
\end{aligned}
$$

and the solution of $\left(y_{s}^{x, t}, z_{s}^{x, t}\right), s \in[t, T]$ of BSDE (1) in the time interval $[t, T]$, with the following special form of $f$ and $\xi: f(y, z, \cdot)=g\left(y, z, x^{x, t}(\cdot)\right)$, $\xi=\Phi\left(x_{T}^{x, t}\right)$, where $x^{x, t}(s), s \in[t, T]$, is an $n$-dimensional diffusion process with the initial condition $(x, t)$. It evolves according to the following SDE

$$
d x_{s}=b\left(x_{s}, s\right) d s+\sigma\left(x_{s}, s\right) d W_{s}, \quad s \in[t, T], \quad x_{t}=x .
$$

The mutual relation is: $u\left(x^{x, t}(s), s\right)=y^{x, t}(s), \sigma^{T} \nabla u\left(x^{x, t}(s), s\right)=z^{x, t}(s)$. Particularly

$$
u(x, t)=y^{x, t}(t), \quad \sigma^{T} \nabla u(x, t)=z^{x, t}(t)
$$

This can be formally checked by simply applying Itô's formula to $u\left(x_{t}, t\right)$. For $m=1$, Peng [1992b] and then Pardoux and Peng [1992] showed that the function $u$ defined by (1.4) is the unique viscosity solution of PDE (1.2) (see Crandall, Ishii and Lions [1992] for the theory of viscosity solution) Pardoux and Peng,[1992] showed that if coefficients are smooth, say $C^{3}$, then $u(x, t)$ defined in (1.4) is the $C^{2,1}$ solution of (1.2) ( $m$ is allowed to be bigger than 1). An interesting problem is, for $m \geq 2$, to find a suitable notion of the solution of PDE of "viscosity type" (1.2) that characterizes $u$ in (1.4). Barles and Lesigne [1997] studied $L^{2}$-space PDE method. El Karoui, Peng and Quenez [1997] introduced a notion of system of quasi linear PDE (1.2). Pardoux and Rao [1997] discussed the viscosity solution for the system of PDE (1.2) with some additional assumptions such as $f^{i}$ does not depends on $z^{j}$ for $i \neq j$. But the general problem still remains an open.

We give two interesting special cases of formula (1.4) (for $m=1$ ). The first one is $f(y, s)=c\left(x_{s}^{x, t}\right) y+g\left(x_{s}^{x, t}\right)$.

The function $u(x, t)$ defined by (1.4) is just the well-known Feynman-Kac formula. It is for this reason that we call (1.4) Nonlinear Feynman-Kac Formula. The second case is the well-known Black \& Scholes formula of option pricing. In this case $f=a y+b z$. [EPQ,1997] gives many further applications of BSDE in finance.

A useful tool in BSDE is Comparison Theorem introduced in Peng [1992b] and improved in Pardoux, Peng [1992], El Karoui, Peng and Quenez [1997]. 
Briefly speaking, if $f$ and $\xi$ are dominated respectively by $f^{\prime}$ and $\xi^{\prime}$, then the corresponding solution $(y, z)$ is also dominated by $\left(y^{\prime}, z^{\prime}\right): y_{t} \leq y_{t}^{\prime}$. Many difficulties concerning the existence theorem in multi-dimensional case $(m \geq 2)$ are due to the lack of this important property.

\section{STOCHASTIC HAMILTON-JACOBI-BELLMAN EQUATIONS}

We consider the following standard control system driven by a control process $\alpha . \in M^{2}(0, T ; U)$ (called an admissible control), its value must be chosen in a given subset $U \subset \mathbf{R}^{k}$. The state variable evolves according to

$$
\begin{aligned}
d X_{s}^{x, t} & =b\left(X_{s}^{x, t}, \alpha_{s}, s\right) d s+\sigma\left(X_{s}^{x, t}, \alpha_{s}, s\right) d W_{s}, \quad s \in[t, T], \\
X_{t}^{x, t} & =x .
\end{aligned}
$$

Some assumptions are needed to ensure the existence and uniqueness of the above stochastic differential equation. We are given the following utility function:

$$
J_{x, t}(\alpha(\cdot))=\mathbf{E}^{\mathcal{F}_{t}}\left\{\int_{t}^{T} f\left(X_{s}^{x, t}, \alpha_{s}\right) d s+\Phi\left(X_{T}^{x, t}\right)\right\},
$$

where $f(x, \alpha, t)$ and $\Phi(x)$ are real-valued functions. Our objective is to maximize the utility function over the set of admissible control. The maximum value is called the value function:

$$
u(x, t)=\operatorname{ess} \sup \left\{J_{x, t}(\alpha(\cdot)) ; \alpha(\cdot) \in M^{2}(0, T ; U)\right\}
$$

A classic situation is when all coefficients $b, \sigma, f$ and $\Phi$ are deterministic functions of $x, \alpha$ and $t$. In this case $u$ is a deterministic function of $(x, t)$. It is the unique viscosity solution of the following Hamilton-Jacobi-Bellman (HJB in short) equation:

$$
\begin{aligned}
-\frac{\partial u}{\partial t} & =\sup _{v \in \mathcal{U}}\left\{\frac{1}{2}\left\langle D^{2} u \sigma(x, \alpha), \sigma(x, \alpha)\right\rangle+\langle D u, b(x, \alpha, t\rangle+f(x, \alpha, t)]\right\}, \\
u(x, T) & =\Phi(x) .
\end{aligned}
$$

But if one of the coefficients are random, e.g., for each $x, \Phi=\Phi(x, \omega)$ is $\mathcal{F}_{\mathcal{T}}$-measurable), the value function $u(x, \cdot)$, for each $x$, becomes an $\mathcal{F}_{t}$-adapted process. It is no longer a solution of HJB equation (2.4). Peng [1992a] pointed out that, provided every thing is "smooth enough", the value function $u(x, \cdot)$ together with $v(x, \cdot)$, another function-valued $\mathcal{F}_{t}$-adapted process, consists the solution of the following backward stochastic PDE (Verification Theorem):

$$
\begin{aligned}
-d u= & \sup _{v \in \mathcal{U}}\left\{\frac{1}{2}\left\langle D^{2} u \sigma(x, \alpha, t), \sigma(x, \alpha, t)\right\rangle+\langle D u, b(x, \alpha, t\rangle\right. \\
& +\langle D v, \sigma(x, \alpha)\rangle+f(x, \alpha, t)\} d t-v(x, t) d W, \\
u(x, T)= & \Phi(x) .
\end{aligned}
$$


This equation is called stochastic HJB equation. The problem is, in most circumstances, one can not expect that the pair $(u, v)(x, \cdot)$ is 'smooth enough'. Peng [1992a] studied the case where the diffusion term is independent of

the control variable: $\sigma=\sigma(x, t)$. An existence and uniqueness theorem has been obtained. A difficult problem is when $\sigma=\sigma(x, \alpha, t)$. Two problems are: (i) To introduce a generalized notion of viscosity solutions of (2.5). (ii) To develop a $C^{2+\alpha}$-theory.

\section{STOCHASTIC MATRIX-VALUED RICCATI EQUATION}

A typical situation of the above optimization is the so-called linear quadratic case, i.e., $U=\mathbf{R}^{\mathbf{k}}, b$ and $\sigma$ are linear functions, $f$ and $\Phi$ are quadratic functions:

$$
\begin{gathered}
b_{t}(x, \alpha)=A_{t} x+B_{t} \alpha, \quad \sigma_{t}(x, \alpha)=C_{t} x+D_{t} \alpha \\
f_{t}(x, \alpha)=-\frac{1}{2}\left[\left\langle R_{t} x, x\right\rangle+\left\langle N_{t} \alpha, \alpha\right\rangle\right], \quad \Phi(x)=-\frac{1}{2}\langle Q x, x\rangle=. .
\end{gathered}
$$

where $A_{t}, B_{t}, C_{t} D_{t}, R_{t}, N_{t}$ and $Q$ are bounded and matrix-valued vith suitable dimentions. $R$., and $Q$ are symmetric and nonnegative. $N$. is strictly positive. It is easy to check that the value function $u$ is also quadratic in $x: u(x, t)=$ $\frac{1}{2}\left\langle Y_{t} x, x\right\rangle$. Consequently, $v$ is also quadratic: $v(x, t)=\frac{1}{2}\left\langle Z_{t} x, x\right\rangle$. Here $Y_{t}$ and $Z_{t}$ are $n \times n$-symmetric matrix-valued processes. $Y_{t}$ is nonnegative. From (14) one can formally write:

$$
-d Y_{t}=F\left(Y_{t}, Z_{t}, t\right) d t-Z_{t} d W_{t}, \quad Y_{T}=Q,
$$

where $F(Y, Z, t)$ is an $n \times n$-valued function:

$$
\begin{aligned}
- & F(Y, Z, t)=A_{t}^{T} Y+Y A_{t}+C_{t}^{T} Z+Z C_{t}+C_{t}^{T} Y C_{t}+R_{t} \\
& -\left(Y B_{t}+Z D_{t}+D_{t} Y C_{t}^{T}\right)\left(N_{t}+D_{t}^{T} Y D_{t}\right)^{-1}\left(Y B_{t}+Z D_{t}+D_{t} Y C_{t}^{T}\right)^{T} . .
\end{aligned}
$$

The problem is to prove the existence and uniqueness of (3.3). When

$D=0$, the problem is solved, see Bismut [1976], Peng [1992a,1993]. The case $n=1, D \neq 0$ is solved ([OT,1997]).

\section{GENERAL MAXIMUM PRINCIPLE UNDER RECURSIVE UTILITIES}

The above stochastic optimization problem can also be treated by the method of stochastic maximum principle of Pontryagin's type. Let $H$ be the "stochastic Hamiltonian" introduced in Bismut [1973] (see also Bensoussan [1981]) defined by $H=\langle p, b(x, \alpha, t)\rangle+\langle q, \sigma(x, \alpha, t)\rangle+f(x, \alpha, t)$. Based on the Bisnut's 'local maximum principle', Peng [1990] obtained the following 'general stochastic 
maximum principle':

$$
\begin{aligned}
\sup _{\alpha \in U} & \left\{H\left(x_{t}, \alpha, p_{t}, q_{t}-P_{t} \sigma\left(x_{t}, \alpha_{t}\right), t\right)-\frac{1}{2}\left\langle P(t) \sigma\left(x_{t}, \alpha\right), \sigma\left(x_{t}, \alpha\right)\right\rangle\right\} \\
\leq & H\left(x_{t}, \alpha_{t}, p_{t}, q_{t}-P_{t} \sigma\left(x_{t}, \alpha_{t}\right), t\right)-\frac{1}{2}\left\langle P(t) \sigma\left(x_{t}, \alpha\right), \sigma\left(x_{t}, \alpha_{t}\right)\right\rangle
\end{aligned}
$$

where the pair $(p ., q.) \in M^{2}(0, T ; \mathbf{R})^{2}$ is the solution of the following linear BSDE

$$
-d p_{t}=\partial_{x} H\left(x_{t}, \alpha_{t}, p_{t}, q_{t}, t\right) d t-q_{t} d W_{t}, \quad p_{T}=\Phi\left(x_{T}\right) .
$$

and $\left(P_{t}, Q_{t}\right)$ solves the following $n \times n$-matrix valued BSDE:

$$
\begin{aligned}
-d P_{t}= & {\left[b_{x}^{T} P+P b_{x}+\sigma_{x}^{T} Q+Q \sigma_{x}+\sigma_{x}^{T} P \sigma_{x}\right.} \\
& \left.+H_{x x}\left(x_{t}, \alpha_{t}, p_{t}, q_{t}, t\right)\right] d t-Q_{t} d W_{t}, \\
P_{T}= & \Phi_{x x}\left(x_{T}\right) .
\end{aligned}
$$

Recently, Duffie, Epstein [1992a,b] and other economists are introduced the notion of recursive utilities in continuous time (see also Duffie, Lions [1992]). El Karoui, Peng and Quenez [1997] give the following general form of recursive utility:

$$
J_{x_{0}, 0}(\alpha(\cdot))=y_{0}
$$

where $y_{0}$ is solved via the following BSDE

$$
-d y_{t}=f\left(x_{t}, \alpha_{t}, y_{t}, z_{t}, t\right) d t-z_{t} d W_{t}, \quad y_{T}=\Phi\left(x_{T}\right) .
$$

It is easy to check that, when $f$ only depends on $(x, \alpha)$ : i.e.,

$f=f(x, \alpha, t)$, then $J(\alpha(\cdot))$ is the classical utility function of type (2.2). Local maximum principle under the recursive utility is given in Peng $[\mathrm{P}, 1993]$ (see also Xu [1994], Wu and Xu [1997]. The corresponding 'global maximum principle' for the case where $f$ depends nonlinearly on $z$ is open, except for some special case (see Peng and Xu [1998]).

\section{EXISTENCE OF BSDE WITH NON LIPSCHITZ COEFFICIENTS}

We return to a general BSDE of form (1.1). Since [PP,1990] many efforts have been made to relax the assumption of the uniform Lipschitz condition imposed on $f$. For 1-dimensional case, i.e., $m=1$, see Lepeltier, San Martin [1996]; Kobylansky [1997] shows that the linear growth condition with respect to $(y, z)$ can be relaxed to the quadratic growth condition provided the prescribed terminal value $y_{T}=\xi$ is bounded (see also [HLS,1998] for more general situation). This is a very interesting result. But the boundness of $\xi$ excludes a very interesting application, i.e., the problem of risk sensitive control (see 
Bensousssan and Nagai[1996], Bielecki and Pliska [1998]). This problem can be naturally formulated as a BSDE with $f$ quadratic in $z$. In this domain one often interested in the situations where $\xi \in L^{2}$. Risk sensitive control problems also indicate a totally open topic in BSDE: the problem of explosions.

Another challenge in BSDE is for multi-dimensional case: $m \geq 2$. The problem of the existence with coefficient $f$ only continuous in $(y, z)$ becomes very hard (a Markov case was treated in Hamadame Lepeltier and Peng [1997]). Similar problem appears in situation of the quadratic growth condition. The techniques that successfully applied in 1-dimensional case fails since the lack of the comparison theorem. Multi-dimensional case has many interesting applications. For example: an important open questions in fining the equilibrium strategies in non-zero-sum differential games is formulated as a BSDE where $f$ is only continuous (see [EPQ,1997], [HLP,1997]). The quadratic growth condition relates to harmonic mappings in differential geometry (see Darling [1995]).

\section{FORWARD-BACKWARD STOCHASTIC DIFFERENTIAL EQUATIONS}

Forward-Backward Stochastic Differential Equations (in short, FBSDE) is derived from many stochastic optimization problems. For fully coupled situation, a local existence and uniqueness result was obtained in Antonelli [1992]

(see also [P,1992c]). The first global existence and uniqueness results, i.e., in an arbitrarily large interval $[0, T]$, was given in Ma and Yong [MY,1995], Ma, Protter and Yong [1994], which is now well known as "four-step-approach". Recently, Pardoux and Tang [1997] also give a new approach.

Here we discuss a different global existence and uniqueness approach given in $\mathrm{Hu}$ and Peng [1994] with a continuation method (see also Peng [1991a]). This approach is generalized in Peng and Wu [1996], Yong [1997] (Bridge Method). Let $b, \sigma$ and $f$ be three $\mathbf{R}^{n}$-valued function of $\zeta=(x, y, z) \in\left(\mathbf{R}^{n}\right)^{3}$. We assume that the mapping $A$ defined by $A(\zeta)=(-f, b, \sigma)(\zeta):\left(\mathbf{R}^{n}\right)^{3} \longmapsto\left(\mathbf{R}^{n}\right)^{3}$ is Lipschitz in $\zeta$ and is monotonic:

$$
\left\langle A(\zeta)-A\left(\zeta^{\prime}\right), \zeta-\zeta^{\prime}\right\rangle \leq-\beta\left\|\zeta-\zeta^{\prime}\right\|^{2}
$$

We also assume that $\Phi$ is Lipschitz in $x$ and is monotonic: $\left\langle\Phi(x)-\Phi\left(x^{\prime}\right), x-\right.$ $\left.x^{\prime}\right\rangle \geq 0$. For each fixed $(x, t) \in \mathbf{R}^{n} \times[0, T]$, we consider the following problem of FBSDE: to find a triple of processes $\left(X_{s}^{x, t}, Y_{s}^{x, t}, Z_{s}^{x, t}\right), s \in[t, T]$, that solves

$$
\begin{aligned}
d X_{s}^{x, t} & =b\left(X_{s}^{x, t}, Y_{s}^{x, t}, Z_{s}^{x, t}\right) d s+\sigma\left(X_{s}^{x, t}, Y_{s}^{x, t}, Z_{s}^{x, t}\right) d W_{s}, \quad X_{t}^{x, t}=x . \\
-d Y_{s}^{x, t} & =f\left(X_{s}^{x, t}, Y_{s}^{x, t}, Z_{s}^{x, t}\right) d s-Z_{s}^{x, t} d W_{s}, \quad Y_{T}^{x, t}=\Phi\left(X_{T}^{x, t}\right) .
\end{aligned}
$$

By $\mathrm{Hu}$ and Peng [1994], this equation has a unique solution. We then can define the following two (deterministic) function of $(x, t)$,

$$
u(x, t)=Y_{t}^{x, t}, \quad v(x, t)=Z_{t}^{x, t} .
$$


If the pair $(u, v)$ is sufficiently smooth, then it is the solution of the following PDE

$$
\begin{aligned}
-\frac{\partial u}{\partial t} & =\frac{1}{2}\left[\sigma \sigma^{T}(x, u, v)\right]_{i j} \frac{\partial^{2} u}{\partial x_{i} \partial x_{j}}+b_{i}(x, u, v) \frac{\partial u}{\partial x_{i}}+f(x, u, v), \\
v(x, t) & =\sigma^{T}(x, u, v) \nabla u, \quad(x, t) \in \mathbf{R}^{n} \times[0, T] .
\end{aligned}
$$

with a Cauchy type of terminal condition: $u(x, T)=\Phi(x)$. An interesting problem is how to find a suitable notion of the solution $(u, v)$ of this PDE that coincides with (6.2). A more general case is when $b, \sigma, f$ and $\Phi$ are random, e.g., for each $x \in \mathbf{R}^{n}, \Phi(x, \omega)$ is $\mathcal{F}_{T}$ measurable. In this situation the pair $(u(x, \cdot), v(x, \cdot))$ defined in $(6.2)$ is an $\left(\mathbf{R}^{n}\right)^{2}$-valued $\mathcal{F}_{t}$-adapted process. The following backward stochastic PDE is then in order:

$$
\begin{aligned}
-d u= & \left\{\frac{1}{2}\left[\sigma \sigma^{T}(x, u, v)\right]_{i j} \frac{\partial^{2} u}{\partial x_{i} \partial x_{j}}+b_{i}(x, u, v) \frac{\partial u}{\partial x_{i}}\right. \\
& \left.+\sigma^{T}(x, u, v) \nabla v+f(x, u, v)\right\} d t-v d W_{t}, \\
v(x, t)= & \sigma^{T}(x, u, v) \nabla u, \quad(x, t) \in \mathbf{R}^{n} \times[0, T] .
\end{aligned}
$$

with the terminal condition: $u(x, T)=\Phi(x)$. The question is to prove the existence and uniqueness of (6.4).

\section{References}

Antonelli, F.(1992): 'Backward-Forward Stochastic Differential Equations" Annals of Applied Probability 3. 777-793 (1993).

Bensoussan, A.,(1981) "Lecture on Stochastic Control", LNM No. 972, Nonlinear Filtering and Stochastic Control.

Bensoussan, A., and Nagai, H., (1996): "Min-Max Characterization of Small Noise Limit on Risk-Sensitive Control" SIAM J. Contr. and Optim., 35(4), 1093-1115.

Barles, G., Lesigne, E., (1997): "SDE, BSDE and PDE", Backward Stochastic Differential Equation, El Karoui, N., Mazliak, L. (Editors), , pp47-82, Longman.

Bielecki, T.R., Pliska S.R., (1998) " Risk Sensitive Dynamic Asset Menagement", to appear in J. Appl. and Optim..

Bismut, J.M. (1973): "Conjugate Convex Functions in Optimal Stochastic Control," J.Math. Anal. Apl., 44, pp.384-404.

Bismut, J.M. (1978): "Contrôle des systèmes linéaires quadratiques : applications de l'intégrale stochastique," Sémin.Proba. XII., Lect. Notes in Mathematics, 649, pp.180-264, Springer.

Black, F., and M.Scholes (1973): "The Pricing of Options and Corporate Liabilities," Journal of Political Economy,3, pp.637-654.

Chen Z., Epstein, L., (1998): "Ambiguity, Risk and Asset Returns in Continuous Time", Preprints. 
Crandall, M.G., Ishii, H., and Lions, P.L., (1992): "User's Guide to Viscosity Solutions of Second Order Partial Differential Equations", Bull. AMS., 27, 1-67.

Darling, R.W.R., (1995): "Constructing Gamma-Martingales with Prescribed Limits, Using Backward SDE", Ann. Prob., 23, 1234-1261.

Duffie, D., and L.Epstein (1992a): "Stochastic Differential Utility," Econometrica, 60, (2), 353-394.

Duffie, D., and L.Epstein (1992b): “ Asset Pricing with Stochastic Differential Utility" , Review of Financial Studies, 5, 411-436.

Duffie, D., J.Ma, and J.Yong (1994): "Black's Consol Rate Conjecture", Ann.Applied probability, 5, 356-382.

El Karoui, N., Mazliak, L. (Editors), (1997): Backward Stochastic Differential Equation, Longman.

El Karoui, N., S.Peng, and M.C.Quenez (1994): "Optimization of Utility Functions" Working Paper, Paris VI University.

Fleming, W.F., and Rishel (1975): Deterministic and Stochastic Control, Springer-Verlag.

Hamadène, S., and Lepeltier, J.P., (1995): "Zero-Sum Stochastic Differential Games and Backward Equations", Systems and Control Letters, 24, 259-263.

Hamadène, S., and Lepeltier, J.P., (1995): "Backward Equations, Stochastic Control and Stochastic Differential Games", Stoch. and Stoch. Reports, 54, 221-231.

Haussmann,U.G., (1976) "General Necessary Conditions for Optimal Con- trol of Stochastic system", Math.Programming study 6.

Hu, Y. and Peng, S., (1995): "Solution of Forward-Backward Stochastic Differential Equations", Prob. and Related Fields, 103, 273-283.

Kobylansky, M.,(1997): BSDEs and PDEs, The Case with Quadratic Growth, Preprint.

Krylov, N.(1980): Controlled Diffusion Processes, New York. Springer Verlag.

Lepeltier, J.P., and San Martin, J., (1996): "Backward Stochastic Differential Equations with Continuous Generators", Proba. and Stat. Letters, to appear.

Li, X., and Tang, Sh., (1995) "General Necessary Conditions for Partially Observed Optimal Stochastic Controls", J. Appl. Prob., 32, 1118-1137.

Ma, J., P.Protter, and J.Yong (1994): "Solving Forward-backward Stochastic Differential Equations Explicitly - a four step Scheme", Proba. Theory Relat. Fields, 98, 339-359 .

Ma, J., and J.Yong (1995): "Solvability of Forward-backward Stochastic Differential Equations and Nodal Set of Hamilton-Jacobi-Bellman Equations" Annals of Mathematics.

Pardoux, E. and S.Peng (1990): "Adapted Solution of a Backward Stochastic Differential Equation", Systems and Control Letters, 14, 55-61. 
Pardoux, E. and S.Peng (1992): “Backward Stochastic Differential equations and Quasilinear Parabolic Partial Differential Equations" Lect. Notes in CIS ,176, 200-217, Springer-Verlag.

Peng, S. (1990): "A General Stochastic Maximum Principle for Optimal Control Problems" SIAM J. Control and Optimization. 28 (4), 966-979.

Peng, S. (1991a): "Probabilistic Interpretation for Systems of Quasilinear Parabolic PDE and applications", Stoch. and Stoch. Reports, 37, 61-74.

Peng, S. (1991b): "A Nonlinear Feynman-Kac Formula and Applications" Proceedings of Symposium of System Sciences and Control theory, Chen 6 Yong ed. 173-184, World Scientific, Singapore.

Peng, S. (1992a): "Stochastic Hamilton-Jacobi-Bellman Equations", SIAM J. Control and Optimization 30 (2), 284-304.

Peng, S. (1992b): "A Generalized Dynamic Programming Principle and Hamilton-Jacobi-Bellman equation", Stochastics, 38, 119-134.

Peng, S. (1993): "Backward Stochastic Differential Equation and It's Application in Optimal Control", Appl. Math. E6 Optim., 27,125-144.

Peng, S. (1997b): "Duplicating and Pricing Contingent Claims in Incomplete Markets", to appear.

Tang, S., (1997): "The Maximum Principle for Partially Observed Optimal Control of Stochastic Differential Equations", SIAM J. Contr. and Optim., to appear.

Tang, S., and Li, X., (1994): "Necessary Conditions for Optimal Control of Stochastic Systems with Random Jumps", SIAM J. Contr. and Optim. 32(5), 1447-1475.

Yong, J., (1997): "Finding adapted solutions of forward-backward stochastic differential equations - method of continuation" Prob. Theory and Rel. Fields, 107, 537-572. 